\title{
DNA Repair in Sea Star Asterias Rubens: Genomic Aspects
}

\section{Leclerc $\mathbf{M}^{*}$}

556 Rue Isabelle Romée, Sandillon, 45640, France

*Corresponding author: Michel Leclerc, 556 Rue Isabelle Romée, Sandillon, 45640,

\section{Short Communication}

Volume 2 Issue 1

Received Date: January 14, 2017

Published Date: January 18, 2017

France, Tel: 0238410209; Email: mleclerc45@gmail.com

\section{Abstract}

In the present paper, we study the DNA repair through the animal kingdom and especially in the sea star system. Rad 51 family, found in Drosophila was shown in sea star genome, so that genes which are usually described in vertebrates.

Keywords: DNA repair genes; Sea star; Invertebrates

\section{Introduction}

Both genetic and biochemical approaches have been used, to study the molecular mechanisms, by which damaged DNA is repaired, in a number of species. The fundamental DNA repair pathways have been functionally conserved for the most part among, prokaryotes, lower eukaryotes and higher eukaryotes. The proteins and protein families, involved, in these repair processes, show high degrees of amino-acid sequence conservation. However, there are also a number of cases in which lack of conservation of particular polypeptides may reveal interesting species specific differences in how certain repair functions are performed.

\section{DNA Methylation}

In mammals, DNA methylation is associated with the regulation of gene expression and the maintenance of the differential state in cell lineages [1]. It is also present in other phylogenetic groups such as some invertebrates: sponges, some insects, sea urchins.

\section{DNA repair in Invertebrates}

The recent completion of the Drosophila genome sequence show the existence of the Rad 51 Family DNA repair.

(Rad 51 named for Saccharomyces cerevisiae: the first member discovered). Two Drosophila Rad 51 have been described [2] there are important in both recombinational DNA repair and meiotic recombination.

\section{Materials and Methods}

Sea stars were obtained from Gothenburg University (Sweden) Sea star Asterias rubens genome has recently been studied [3] in immunized and non-immunized sea stars to HRP (Horse-radish peroxydase).

\section{Results}

We find Rad 51 Family DNA repair and also what is original and not yet described, genes which are found in vertebrates exclusively:

In non-immunized sea star genome ("control")

Control: TR38504|c0_g1_i1 sp|Q64267|XPA_MOUSE DNA repair protein complementing XP-cells homolog OS=Mus; musculus; $\mathrm{GN}=\mathrm{Xpa} ; \mathrm{PE}=1 \mathrm{SV}=2$.

Control: Contig11913 sp|P23475|XRCC6_MOUSE X-ray repair cross-complementing protein $6 \mathrm{OS}=$ Mus musculus; $\mathrm{GN}=\mathrm{Xrcc} 6 ; \mathrm{PE}=1 \mathrm{SV}=5$.

Control: Contig7953 sp|P27641|XRCC5_MOUSE X-ray repair cross-complementing protein 5 OS=Mus musculus;GN=Xrcc5; PE=2 SV=4.

We find similar results in immunized sea star genome. 


\section{Cell \& Cellular Life Sciences Journal}

\section{Interpretation-Discussion}

These genes, in mouse, are mainly used in cellular response to X-Ray, to gamma radiation, in Vertebrates.It is surprising to find them in an ancestral invertebrate: the sea star Asterias rubens: The sea star remains an enigmatic animal. Another explication can be performed: These genes named, in mouse: Xrcc6 and Xrrc5 play a role in the immune process in which immune receptor $\mathrm{V}, \mathrm{D}$ and $\mathrm{J}$, or $\mathrm{V}$ and $\mathrm{J}$ gene segments, depending on the specific receptor are recombined within a single locus utilizing the conserved heptamer and nonomer recombination signal sequence (RSS) regional genes (V,D,J) used to generate Immunoglobulin molecules. This last aspect of sea star genomic studies corroborates the existence of the sea star primitive antibody.

\section{References}

1. Riggs AD (1990) DNA methylation and late replication probably aid cell memory, and type I DNA reeling could aid chromosome folding and enhancer function. Philos Trans R Soc Lond B Biol Sci 326(1235): 285-297.

2. McKee BD, Bruce D, Xiao-jia Ren, Chia-sin Hong (1996) ArecA-like gene in Drosophila melanogaster that is expressed at high levels in female but not male meiotic tissues Chromosoma 104(7): 479-488.

3. Leclerc M, Sam D, Olga Ortega-Martinez, Bodil HK, Björn R (2011) Evidence of Kappa genes in the seastar Asterias rubens (Echinoderma) Immunol Lett 138(2): 197-198. 